\title{
Évaluations dosimétriques de l'exposition potentielle liée à l'accumulation naturelle d'uranium et de thorium dans les sables de certaines plages du littoral de Camargue
}

\author{
A. THOMASSIN ${ }^{1}$, C. HUET ${ }^{2}$, I. CLAIRAND ${ }^{2}$, E. BLANCHARDON ${ }^{3}$, \\ L. POURCELOT ${ }^{4}$, R. GURRIARAN ${ }^{5}$
}

(Manuscrit reçu le 29 juin 2007, accepté le 11 novembre 2007)

RÉSUMÉ En 2000, des flux de rayonnement anormalement élevés ont été détectés sur certaines plages du littoral camarguais. Les sables du littoral camarguais ont alors fait l'objet d'une caractérisation radiologique montrant des activités moyennes de quelques dizaines de $B q ~ k g^{-1}$ en uranium-238 et thorium-232 d'une part, et l'existence de points chauds à plus de $1000 \mathrm{~Bq} \mathrm{~kg}^{-1} \mathrm{~d}$ 'autre part ; voire plus sur deux plages : l'une située à l'Est du phare de Beauduc, l'autre située à l'Est du phare de l'Espiguette. Cette radioactivité, d'origine naturelle, est responsable de l'exposition des personnes fréquentant ces deux dernières plages relativement isolées, mais néanmoins accessibles à tout public. L'Institut de radioprotection et de sûreté nucléaire a réalisé une évaluation des doses susceptibles d'être reçues par des personnes fréquentant ces deux dernières plages, en se basant sur des mesures et des prélèvements effectués in situ, en établissant expérimentalement la solubilité des sables dans les fluides gastrique et intestinal, et en formulant des hypothèses sur la fréquentation des lieux (scénarios). La dose annuelle maximale ainsi évaluée est de l'ordre de $0,8 \mathrm{mSv}$ pour des adultes et $0,5 \mathrm{mSv}$ pour les enfants ; valeurs vraisemblablement très prudentes dans lesquelles la dose par exposition externe prédomine largement.

ABSTRACT Dosimetric assessment of the exposure due to natural accumulation of uranium and thorium in sands of some beaches of Camargue.

In 2000, abnormally high ray fluxes have been detected on some beaches of Camargue. Then, sands of these beaches have been studied from a radiological point of view, showing on the one hand mean levels of uranium-238 and thorium-232 activity about some tens of $\mathrm{Bq} \mathrm{\textrm {kg } ^ { - 1 }}$, and on the other hand some hot spots with more of $1000 \mathrm{~Bq} \mathrm{~kg}$; and even more on two beaches: one to the east of the Beauduc lighthouse, the other to the east of Espiguette lighthouse. This radioactivity, of natural origin, is responsible of exposure of the public frequenting these two secluded beaches, but nevertheless open to everybody. The Institute for radiological protection and nuclear safety has carried out a dose assessment for people likely to

\footnotetext{
1 IRSN, Unité d'expertise en radioprotection des travailleurs et de la population, DRPH/SER/UETP, B.P. 17, 92262 Fontenay-aux-Roses, France.

IRSN, Laboratoire de dosimétrie des rayonnements ionisants, DRPH/SDE/LDRI, B.P. 17, 92262 Fontenay-aux-Roses, France.

IRSN, Laboratoire d'évaluation de la dose interne, DRPH/SDI/LEDI, B.P. 17, 92262 Fontenay-aux-Roses, France.

IRSN, Laboratoire d'étude radioécologique du milieu continental et marin, DEI/SESURE/LERCM, B.P. 3, 13115 SaintPaul-Lez-Durance, France.

5 IRSN, Laboratoire de mesure de la radioactivité dans l'environnement, DEI/STEME/LMRE, Bois des Rames, bâtiment 501, 91400 Orsay, France.
} 
frequent these beaches, taking into account local measurements and samples, in establishing the solubility of sands in the gastric and intestinal fluids, and with assumptions for site frequentation (scenarios). The maximal annual effective dose is about $0.8 \mathrm{mSv}$ for adults and about $0.5 \mathrm{mSv}$ for children; very wise values, in which external exposure widely predominates.

Keywords: natural radioactivity / uranium / thorium / sand / dose assessment

\section{Introduction}

Comme de nombreuses plages de par le monde, celles de Camargue présentent ponctuellement des zones de fortes activités en uranium et en thorium. En 2000, la découverte de deux points chauds principaux, situés à l'Est des phares de l'Espiguette et de Beauduc, a conduit l'Institut de radioprotection et de sûreté nucléaire (IRSN) à une expertise des sites concernés et de leurs sables, de manière à apprécier le risque radiologique associé à la fréquentation de ces plages. Le travail d'expertise réalisé est détaillé dans le rapport Thomassin et al. (2007).

Après une caractérisation radiologique des plages et des sables concernés, une estimation dosimétrique basée sur des scénarios raisonnablement prudents de la fréquentation des lieux par le public est proposée.

\section{Caractérisation de la radioactivité des sables et des plages}

Une cartographie de la radioactivité du littoral du golfe du Lion et de la Camargue (prélèvements in situ et mesures héliportées) montre que les activités des sables en uranium et thorium fluctuent dans une large gamme: de quelques dizaines à quelques centaines de $\mathrm{Bq} \mathrm{kg}^{-1}$. En certains points du littoral camarguais - est des phares de l'Espiguette et de Beauduc, l'activité des sables peut atteindre plusieurs milliers de $\mathrm{Bq} \mathrm{kg}^{-1}$, sur des bandes de plage d'une centaine de mètres de long environ. Les activités du thorium-232 et de l'uranium-238, mesurées par spectrométrie $\gamma$ dans l'échantillon le plus actif de la plage de Beauduc, sont de l'ordre de 3400 et $2300 \mathrm{~Bq} \mathrm{~kg}^{-1}$ respectivement.

Sur la plage de Beauduc où les activités en uranium et en thorium des sables sont les plus élevées, les débits de kerma photonique maximaux observés dans l'air sont de $1,47 \mu \mathrm{Gy} \mathrm{h}^{-1}$ à $1 \mathrm{~m}$ du sol et de $2,14 \mu \mathrm{Gy} \mathrm{h}^{-1}$ au sol (15 à 20 fois le fond moyen naturel); la fluence d'électrons maximale observée au sol y est de $0,57 \beta \mathrm{s}^{-1} \mathrm{~cm}^{-2}$.

Une étude granulométrique montre que la radioactivité est portée par des grains fins de sable, riches en minéraux lourds (zircons et apatites). Ces minéraux proviennent des massifs granitiques du bassin rhodanien. Ils sont charriés par le fleuve et redistribués sur la côte camarguaise. 


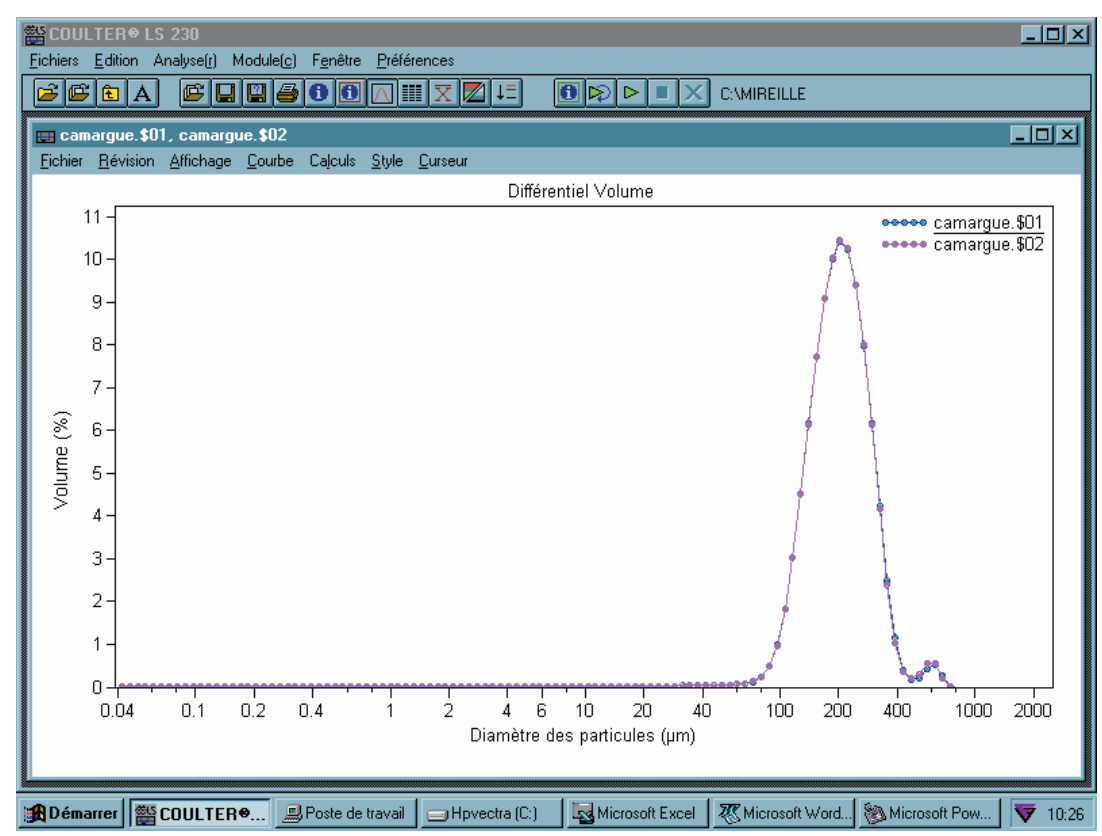

Figure 1 - Distribution volumique des sables en fonction du diamètre des grains.

Volumic distribution of sands according to grain diameter.

La granulométrie des deux échantillons de sable les plus actifs de la plage de Beauduc (étudiée par compteur de particules et par tamisage) est illustrée par la figure 1. Cette figure montre que les grains de sable de diamètre compris entre 100 et $300 \mu \mathrm{m}$ contribuent majoritairement au volume du sable. Le sable analysé contient très peu de particules inhalables (taille inférieure à $50 \mu \mathrm{m}$ ). Seules $0,002 \%$ des particules ont une taille comprise entre 20 et $50 \mu \mathrm{m}$ et un pourcentage négligeable de particules ont un diamètre inférieur à $20 \mu \mathrm{m}$.

Par ailleurs, la concentration dans l'air des grains de sables, mesurée par vent fort (plus de $100 \mathrm{~km} \mathrm{~h}^{-1}$ - situation extrême) est comprise entre 5 et $23 \mathrm{mg} \mathrm{m}^{-3}$. Dans ces conditions, les activités volumiques dans l'air sont comprises entre 4,8 et $17 \mathrm{mBq} \mathrm{m}^{-3}$ pour le thorium-234 (descendant de l'uranium-238), et entre 8,3 et $30 \mathrm{mBq} \mathrm{m}^{-3}$ pour l'actinium-228 (descendant du thorium-232). La quantité d'uranium et de thorium susceptible d'être présente dans l'air des lieux étant très faible d'une part, la fraction inhalable du sable étant très faible (voire négligeable) d'autre part, les voies d'atteinte par exposition à l'atmosphère des lieux (externe par irradiation et interne par inhalation) ne sont pas retenues. 


\section{2. Évaluations dosimétriques}

\subsection{Scénarios}

À défaut d'une observation précise des populations fréquentant les lieux, l'évaluation dosimétrique de l'exposition du public est faite en considérant des scénarios simulant a priori le comportement de personnes sur les lieux pendant un an.

La dose efficace est calculée selon les modalités de l'arrêté du $1^{\text {er }}$ septembre 2003 (JO, 2003), à savoir la somme de celles résultant des expositions externe et interne aux rayonnements ionisants.

Les lieux n'étant pas des lieux de pêche d'une part, et aucune mesure dans les indicateurs marins des environs n'ayant montré des teneurs en uranium et thorium significatives d'autre part, l'exposition interne par ingestion de produits de la mer n'est pas retenue. En conséquence, seules sont retenues les voies d'atteinte d'exposition externe à la surface des lieux contaminés (sable) et d'exposition interne par ingestion par inadvertance de sable.

Les quatre scénarios suivants sont considérés :

- un premier scénario local d'un adulte ou adolescent (13-17 ans) fréquentant les lieux pour les loisirs, à raison de 60 visites par an de 4 heures chacune,

- un deuxième scénario de villégiature pour des adultes ou adolescents utilisant les quelques cabanes de pêcheurs des environs pour des loisirs de type weekend, à raison de 18 visites par an de 2 jours chacune,

- un troisième scénario d'une famille locale fréquentant les lieux pour les loisirs, à raison de 13 visites par an de 6 heures chacune,

- un quatrième scénario d'une famille de touristes fréquentant les lieux pour les loisirs de leurs vacances, soit 30 visites de 8 heures.

Les personnes de la population sont regroupées en classes d'âge, selon le découpage de la Commission internationale de protection radiologique (CIPR); à savoir : nourrisson, enfant de 1 à 2 ans, enfant de 3 à 7 ans, enfant de 8 à 12 ans, adolescent de 13 à 17 ans et adulte (ICRP, 1994).

La distribution horaire des différentes activités sur les lieux (sommeil, repos assis ou couché sur le sable, exercice léger ou intense) varie en fonction du scénario et de la classe d'âge. Pour les scénarios locaux, famille locale et famille touriste, les distributions horaires d'activités sont arbitraires, mais ont été choisies sur la base du raisonnablement envisageable. Pour le scénario de villégiature, la distribution horaire sommeil / repos (lecture + bains de soleil) / exercice léger / 
TABLEAU I

Distribution horaire des activités (h). Activity (h).

\begin{tabular}{|c|c|c|c|c|c|c|c|}
\hline Scénario & Activité (position) & $\frac{\dddot{U}}{E}$ & 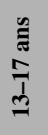 & 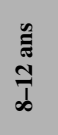 & $\begin{array}{l}\stackrel{0}{\Xi} \\
\text { ஸे } \\
\text { m. }\end{array}$ & 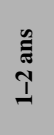 & 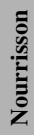 \\
\hline \multirow{4}{*}{$\begin{array}{c}\text { Local } \\
\text { (une visite) }\end{array}$} & repos (assise) & 2 & 2 & & & & \\
\hline & repos (couchée) & 1 & 1 & & & & \\
\hline & exercice léger (debout) & 0,5 & 0,5 & & & & \\
\hline & exercice intense (debout) & 0,5 & 0,5 & & & & \\
\hline \multirow{5}{*}{$\begin{array}{l}\text { Villégiature } \\
\text { (un jour) }\end{array}$} & sommeil* & 8,5 & 10 & & & & \\
\hline & repos (assise) & 3,5 & 3,5 & & & & \\
\hline & repos (couchée) & 2 & 2 & & & & \\
\hline & exercice léger (debout) & 9,75 & 7,5 & & & & \\
\hline & exercice intense (debout) & 0,25 & 1 & & & & \\
\hline \multirow{4}{*}{$\begin{array}{c}\text { Famille locale } \\
\text { (une visite) }\end{array}$} & repos (assise) & 2 & 2 & 2 & 2 & 1,5 & 0 \\
\hline & repos (couchée) & 2 & 2 & 2 & 2 & 3,5 & 4 \\
\hline & exercice léger (debout) & 1,5 & 1,5 & 1,5 & 2 & $1 * *$ & 2 \\
\hline & exercice intense (debout) & 0,5 & 0,5 & 0,5 & 0 & 0 & 0 \\
\hline \multirow{4}{*}{$\begin{array}{l}\text { Famille touriste } \\
\text { (une visite) }\end{array}$} & repos (assise) & 2 & 2 & 2 & 3 & 3 & 0 \\
\hline & repos (couchée) & 3 & 3 & 3 & 3 & 4 & 6 \\
\hline & exercice léger (debout) & 2 & 2 & 2 & 2 & $1 * *$ & 2 \\
\hline & exercice intense (debout) & 1 & 1 & 1 & 0 & 0 & 0 \\
\hline
\end{tabular}

exercice intense est déduite de la publication 66 de la CIPR (ICRP, 1994), car la durée d'une visite excède une journée ; par contre, la distribution horaire repos (lecture) / repos (bain de soleil) est arbitraire. Le tableau I présente les valeurs des différents paramètres de ces scénarios. Pour chacun d'entre eux, chaque ligne du tableau correspond à une activité sur les lieux et précise la durée retenue pour cette activité pour les différentes personnes considérées dans le scénario ; la position (couchée, assise ou debout) est précisée entre parenthèses pour chaque activité.

Relativement à l'ingestion par inadvertance de sable, les valeurs moyennes d'incorporation quotidienne retenue par les experts du Groupe radioécologie Nord-Cotentin (GRNC, 1999) sont retenues ; à savoir $1,4 \mathrm{mg} \mathrm{j}^{-1}$ pour l'adulte et l'adolescent, 2,8 $\mathrm{mg} \mathrm{j}^{-1}$ pour l'enfant de 8 à $12 \mathrm{ans}, 5,5 \mathrm{mg} \mathrm{j}^{-1}$ pour l'enfant de 3 à 7 ans, $14 \mathrm{mg} \mathrm{j}^{-1}$ pour l'enfant de 1 à 2 ans. 


\subsection{Calcul de la dose par exposition externe}

Pour l'exposition aux photons, la dose efficace est calculée avec les coefficients de conversion de la publication de Saito et al. (1998) qui permettent de convertir une valeur de kerma dans l'air (en gray) en une dose efficace (en sievert) pour certains radionucléides présents à l'état naturel dans le sol et en fonction de la position de la personne exposée. Ces coefficients de conversion variant peu entre l'uranium238 et le thorium-232 d'une part, et variant peu entre les positions couchée sur le dos et couchée sur le ventre d'autre part, les valeurs de 0,7 et $0,77 \mathrm{~Sv} \mathrm{~Gy}^{-1}$ sont retenues pour ce coefficient de conversion respectivement pour les positions debout et couchée ; cette dernière valeur est également utilisée pour la position assise.

Pour l'exposition aux électrons, la valeur maximale du coefficient de conversion de fluence électronique en dose absorbée à la peau de la publication 74 de la CIPR (ICRP, 1996) est retenue, soit $171 \mathrm{pGy} \mathrm{cm}^{2}$ pour des électrons de $0,6 \mathrm{MeV}$.

La dose due à l'exposition externe aux rayonnements des sables est évaluée à partir des valeurs maximales observées sur la plage de Beauduc pour les débits de kerma photonique dans l'air et pour la fluence électronique au sol.

\subsection{Calcul de la dose par exposition interne}

Afin d'évaluer la dose due à l'exposition interne par ingestion de sable par inadvertance, la solubilité du sable dans les fluides gastrique et intestinal est établie expérimentalement (Frelon et al., 2007). L'expérience montre que l'équilibre séculaire des deux familles radioactives (uranium-238 et thorium-232) n'est pas modifié par la digestion gastrique et intestinale. La fraction absorbée $\left(f_{A}\right)$ des radionucléides de la famille radioactive de l'uranium-238 est de 0,0037 pour une personne à jeun et 0,005 pour une personne après son repas ; la fraction absorbée des radionucléides de la famille radioactive du thorium-232 est de 0,0014 pour une personne à jeun et 0,0007 pour une personne après son repas.

La dose due à l'exposition interne est évaluée à partir des incorporations quotidiennes (Sect. 2.1.), des activités de l'échantillon cité précédemment (Sect. 1.), et des fractions absorbées pour l'uranium-238 et le thorium-232. Les calculs sont réalisés avec le logiciel DCAL (Eckerman et al., 2001). DCAL a été développé par le Laboratoire Nationale d'Oak Ridge (États-Unis d'Amérique) pour calculer, en particulier, les coefficients de dose efficace engagée résultant de l'incorporation d'un radionucléide conformément aux méthodes décrites dans les publications 67, 69 et 71 de la CIPR (ICRP, 1995a, 1995b, 1993). Un coefficient 
de dose par unité d'activité ingérée a été calculé pour chaque radionucléide des familles de l'uranium-238 et du thorium-232 et chaque classe d'âge, en tenant compte de la valeur de $f_{A}$ déterminée expérimentalement. Les doses dues à l'ingestion de chaque radionucléide ont ensuite été calculées en multipliant le coefficient de dose par l'incorporation prévue dans le scénario d'exposition, et additionnées en supposant l'équilibre séculaire atteint. Cette hypothèse d'équilibre séculaire est confirmée par la comparaison des mesures de l'activité de radionucléides situés en début et en fin des chaînes de désintégration (Louvat et al., 2000 ; Pourcelot et al., 2003). Enfin, la dose due aux radionucléides apparus par transformation nucléaire dans le corps humain est prise en compte par les modèles dosimétriques de la CIPR et par DCAL.

La dose due aux isotopes du radon piégés dans le sable ne peut pas être calculée à l'aide des modèles des publications 67 et 69 de la CIPR. Elle n'est donc pas calculée ici. Cependant, l'étude de Khursheed (2000) suggère que sa contribution à l'exposition interne globale dans les scénarios étudiés peut être négligée.

\subsection{Résultats}

Le tableau II présente les doses efficaces annuelles dues à l'exposition externe (valeur en gras) et à l'exposition interne (première valeur au dessous pour une ingestion de sable à jeun, seconde valeur au dessous pour une ingestion de sable après un repas).

Pour les adultes et les adolescents, la dose efficace annuelle maximale est de l'ordre de $0,8 \mathrm{mSv}$ pour le scénario villégiature ; pour les enfants, la dose efficace annuelle maximale due à l'exposition externe est de l'ordre de $0,5 \mathrm{mSv}$ pour le

TABLEAU II

Doses efficaces annuelles (mSv).

Annual effective doses (mSv).

\begin{tabular}{|c|c|c|c|c|c|c|}
\hline Scénario & Adulte & 13-17ans & 8-12 ans & 3-7 ans & 1-2 ans & Nourrisson \\
\hline Local & $\begin{array}{c}\mathbf{0 , 4 2} \\
0,0005 \\
0,0006\end{array}$ & $\begin{array}{c}\mathbf{0 , 4 2} \\
0,0006 \\
0,0006\end{array}$ & & & & \\
\hline Villégiature & $\begin{array}{c}\mathbf{0 , 7 7} \\
0,0003 \\
0,0003\end{array}$ & $\begin{array}{c}\mathbf{0 , 7 1} \\
0,0004 \\
0,0004\end{array}$ & & & & \\
\hline Famille locale & $\begin{array}{c}\mathbf{0 , 1 3} \\
0,0001 \\
0,0001\end{array}$ & $\begin{array}{c}\mathbf{0 , 1 3} \\
0,0001 \\
0,0001\end{array}$ & $\begin{array}{c}\mathbf{0 , 1 3} \\
0,0003 \\
0,0003\end{array}$ & $\begin{array}{c}\mathbf{0 , 1 3} \\
0,0007 \\
0,0008\end{array}$ & $\begin{array}{c}\mathbf{0 , 1 6} \\
0,0026 \\
0,0028\end{array}$ & $\begin{array}{c}0,15 \\
- \\
-\end{array}$ \\
\hline $\begin{array}{l}\text { Famille } \\
\text { touriste }\end{array}$ & $\begin{array}{c}\mathbf{0 , 3 9} \\
0,0003 \\
0,0003\end{array}$ & $\begin{array}{c}\mathbf{0 , 3 9} \\
0,0003 \\
0,0003\end{array}$ & $\begin{array}{c}\mathbf{0 , 3 9} \\
0,0007 \\
0,0007\end{array}$ & $\begin{array}{c}\mathbf{0 , 4 2} \\
0,0017 \\
0,0018\end{array}$ & $\begin{array}{c}\mathbf{0 , 4 7} \\
0,0060 \\
0,0064\end{array}$ & $\begin{array}{c}0,47 \\
- \\
-\end{array}$ \\
\hline
\end{tabular}


scénario famille touriste. Dans tous les cas, la dose par exposition interne est négligeable. La dose efficace annuelle étant due à l'exposition externe, les valeurs obtenues reflètent principalement le temps passé sur les lieux, la sensibilité à la classe d'âge étant secondaire.

À titre indicatif, 17 mois de résidence à Paris ou 9 mois de résidence dans le Limousin conduisent à une dose de l'ordre du millisievert par exposition aux rayonnements d'origines tellurique et cosmique (Thomassin et al., 2007). Bien que calculées de manière pénalisante, les doses obtenues ne sont ni négligeables ni importantes ; toutefois, compte tenu qu'elles sont du même ordre de grandeur que celles reçues en moyenne par la population française par exposition aux rayonnements telluriques (LVRE, 2007), elles n'appellent pas de dispositions particulières de la part des pouvoirs publics.

\section{Conclusion}

L'existence d'un excès de radioactivité naturelle sur deux plages de Camargue, à l'Est des phares de l'Espiguette et de Beauduc a conduit l'IRSN à réaliser une évaluation dosimétrique, en envisageant différents scénarios pour la fréquentation des lieux.

À l'aide des mesures et de prélèvements effectués in situ d'une part, et d'expérimentation sur la solubilité des sables dans les fluides gastrique et intestinal d'autre part, l'IRSN évalue la dose annuelle maximale de l'ordre de $0,8 \mathrm{mSv}$ pour des adultes et $0,5 \mathrm{mSv}$ pour les enfants ; valeurs vraisemblablement très prudentes dans lesquelles la dose par exposition externe prédomine largement, et pour lesquelles aucune disposition ne paraît nécessaire de la part des pouvoirs publics.

\section{RÉFÉRENCES}

Eckerman K.F., Leggett R.W., Cristy M., Nelson C.B., Ryman J.C., Sjoreen A.L., Ward R.C. (2001) DCAL: User's Guide to the DCAL System. Report ORNL/TM-2001/190, Oak Ridge National Laboratory, United States of America.

Frelon S., Chazel V., Tourlonias E., Blanchardon E., Bouisset P., Pourcelot L., Paquet F. (2007) Risk assessment after internal exposure to black sand from Camargue, Radiat. Prot. Dosim. (sous presse).

GRNC (1999) Groupe radioécologie Nord-Cotentin, Volume 4 - Estimation des doses et du risque de leucémie associé. Rapport détaillé du GT4, juillet 1999.

ICRP Publication 66 (1994) Human Respiratory Tract Model for Radiological Protection, Ann. ICRP $24(1 / 3)$.

ICRP Publication 67 (1993) Age-dependent Doses to Members of the Public from Intakes of Radionuclides: Part 2, Ingestion Dose Coefficients, Ann. ICRP 23 (3/4).

ICRP Publication 69 (1995a) Age-dependent Doses to Members of the Public from Intakes of Radionuclides: Part 3, Ingestion Dose Coefficients, Ann. ICRP 25 (1). 
ICRP Publication 71 (1995b) Age-dependent Doses to Members of the Public from Intake of Radionuclides: Part 4, Inhalation Dose Coefficients, Ann. ICRP 25 (3-4).

ICRP Publication 74 (1996) Conversion Coefficients for use in Radiological Protection against External Radiation, Ann. ICRP 26 (3/4).

JO (2003) Arrêté du $1^{\text {er }}$ septembre 2003 définissant les modalités de calcul des doses efficaces et des doses équivalentes résultant de l'exposition des personnes aux rayonnements ionisants, JO $\mathrm{n}^{\circ} 262$ du 13 novembre 2003, p. 19326.

Khursheed A. (2000) Doses to systemic tissue from radon gas, Radiat. Prot. Dosim. 88, 171-181.

Louvat D., Calmet D., Arnaud M., Thebault H., Charmasson S., Rommens C., Bouisset P., Barker E. (2000) Niveau radiologique des dépôts sédimentaires récents localisés sur les plages du littoral camarguais, Rapport IPSN DPRE/SERNAT/2000-09, IRSN.

LVRE (2007) Bilan de l'état radiologique de l'environnement français en 2005 - Synthèse des résultats des réseaux de surveillance de l'IRSN, Rapport DEI/SESURE n 2007-30, IRSN (2007).

Pourcelot L., Barker E., Carpena J., Charmasson S., Gauthier-Lafaye F., Louvat D., Marquet J., Pupin J.-P., Vassas C., Vella C. (2003) Radioactivité des sables du littoral camarguais : origine et évolution, $1^{\text {er }}$ état d'avancement - juin 2003, Rapport IRSN DEI/SESURE/2003-01.

Saito K., Petoussi-Henss N., Zankl M. (1998) Calculation of the effective dose and its variation from environmental gamma ray sources, Health Phys. 74, 698-706.

Thomassin A. et al. (2007) Évaluations dosimétriques de l'exposition potentielle liée à l'accumulation naturelle d'uranium et de thorium dans les sables de certaines plages du littoral de Camargue, Rapport IRSN 2007-01, IRSN. 